\title{
14T Full Adder in 125nm CMOS technology for FFT applications using piecewise constant interpolation $f(x)=2.0$
}

G.Naveen Balaji' ${ }^{1}$, P.Malini'2 ${ }^{2}$ T.Poovika ${ }^{3}$, P.Shanmugavadivu ${ }^{4}$, J.Jayageetha ${ }^{5}$

2,3,4- PG Scholar, Department of Electronics and Communication Engineering, SNS College of Technology, Coimbatore -35, India

${ }_{1,5-}$ Assistant Professor, Department of Electronics and Communication Engineering, SNS College of Technology, Coimbatore - 35, India

1,2,3,4-malinittp1995@gmail.com,ecepoovi@gmail.com,shanmugavadivu61@gmail.com,yoursgnb@gmail.com, jayageethajaganathan@gmail.com

Correspondence Author: G.Naveen Balaji. PG Scholar, Department of Electronics and Communication Engineering, SNS College of Technology, Coimbatore -35, India

Received date: 22 June 2018, Accepted date: 16 August 2018, Online date: 8 September 2018

Copyright: (c) 2018 G.Naveen Balaji et al. This is an open-access article distributed under the terms of the Creative Commons Attribution License, which permits unrestricted use, distribution, and reproduction in any medium, provided the original author and source are credited.

\begin{abstract}
Background: Full adders play an important role in applications such as digital signal processors (DSP) architectures and microprocessors, according to the concept of adding two numbers, which tends to be its main task, it also participates in many other useful operations such as subtraction, multiplication, division, address calculation, etc. Objective: Modeling and simulation of a $14 \mathrm{~T}$ full adder in static and dynamic style using $125 \mathrm{~nm}$ technology. Results: The window function for each (static, dynamic, 14T) design styles is carried out for the interpolation 2 with the $\mathrm{N}$ value 10 is performed. The power analysis performed is found to have a max power of 3.8 and min power of 1.7. The FFT transformation of an image is performed and the corresponding noise factor and the strength is calculated. Conclusion: By the use of Fast Fourier Transformation (FFT), the transformation used in each window allows to evaluate the power at various interpolation rate. Thus, the scope lies in a reduced power consumption.
\end{abstract}

Key words: Adder, CMOS, static, dynamic, $125 \mathrm{~nm}$ technology, interpolation, FFT, power analysis.

\section{INTRODUCTION}

Digital signal processing (DSP) involves the processing of digital signals that comprises the use of computers or more specialized digital signal processors that performs a wide variety of signal processing operations. The signals are processed in a sequence of numbers that represent samples of a continuous variable in various domain such as time, space, or frequency. The two important subfields of signal processing are digital signal processing and analog signal processing. Some of the Digital Signal Processing applications include audio and speech processing, sonar, radar and other sensor array processing etc.

II $\quad$ 14T Full Adder

The designed 14T Full adder has three inputs and two outputs. The outputs S and Cout are computed using the following expressions.

$\mathrm{S}=\mathrm{A} \oplus \mathrm{A} \oplus \mathrm{C} "$

Cout=A.B + B.C +C.A........... (2)

The 1-bit CMOS full adder cell is shown in Fig1. The 1-bit full adder cell consists of 14 transistors. Designing of logic gates with such large no of transistors makes the design area inefficient, increases power consumption, reduction in speed [1]. Dynamic power dissipation and larger delay is caused due to large PMOS transistors in pull up network that results in high input capacitances. The schematic diagram of a 14T full adder is shown in Fig 2.

Fig 1: Logic diagram of Full adder

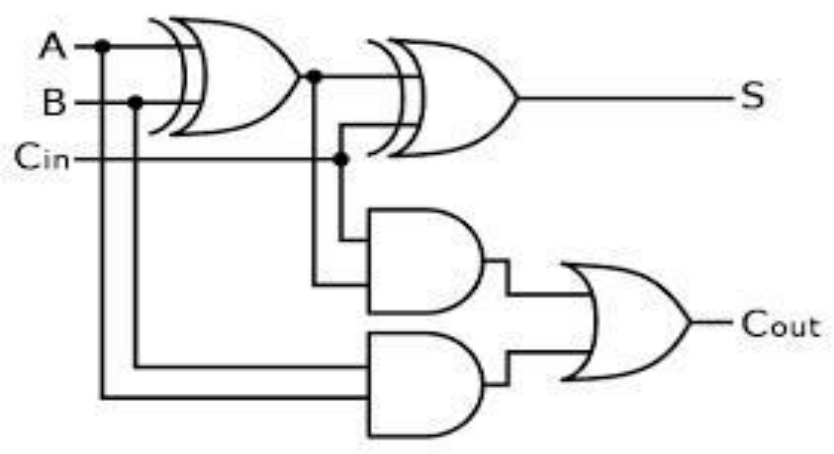


Table 1. Truth Table Full Adder

\begin{tabular}{|c|c|c|c|c|}
\hline A & B & C & SUM & COUT \\
\hline 0 & 0 & 0 & 0 & 0 \\
\hline 0 & 0 & 1 & 1 & 0 \\
\hline 0 & 1 & 0 & 1 & 0 \\
\hline 0 & 1 & 1 & 0 & 1 \\
\hline 1 & 0 & 0 & 1 & 0 \\
\hline 1 & 0 & 1 & 0 & 1 \\
\hline 1 & 1 & 0 & 0 & 1 \\
\hline 1 & 1 & 1 & 1 & 1 \\
\hline
\end{tabular}

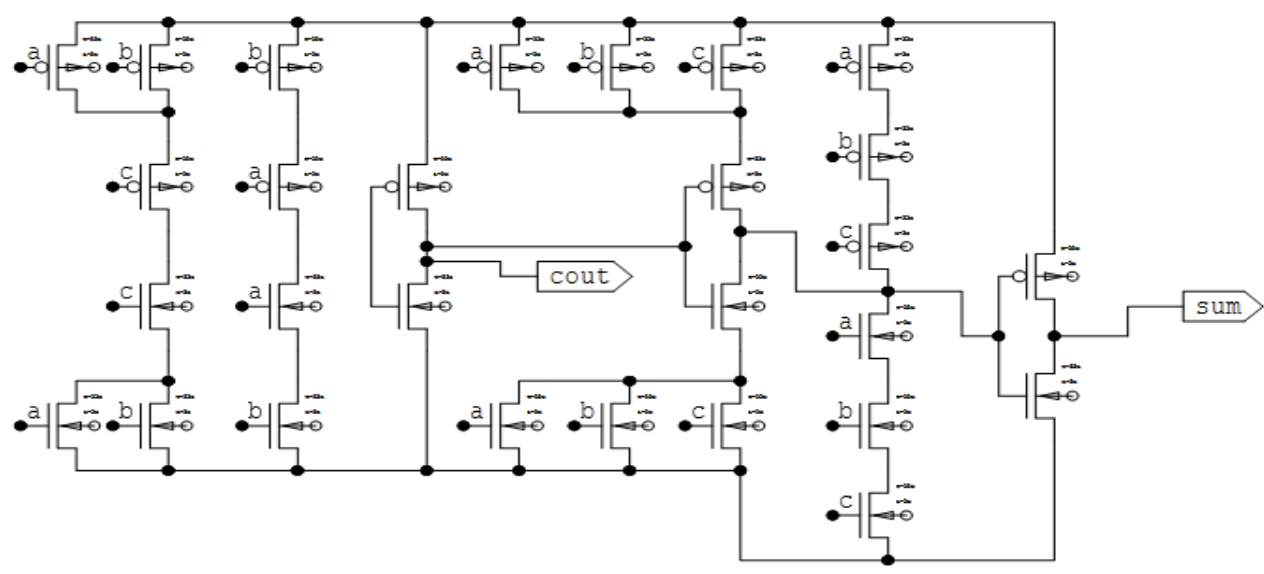

Fig 2: Schematic diagram for 14T full adder

\section{B. Netlist}

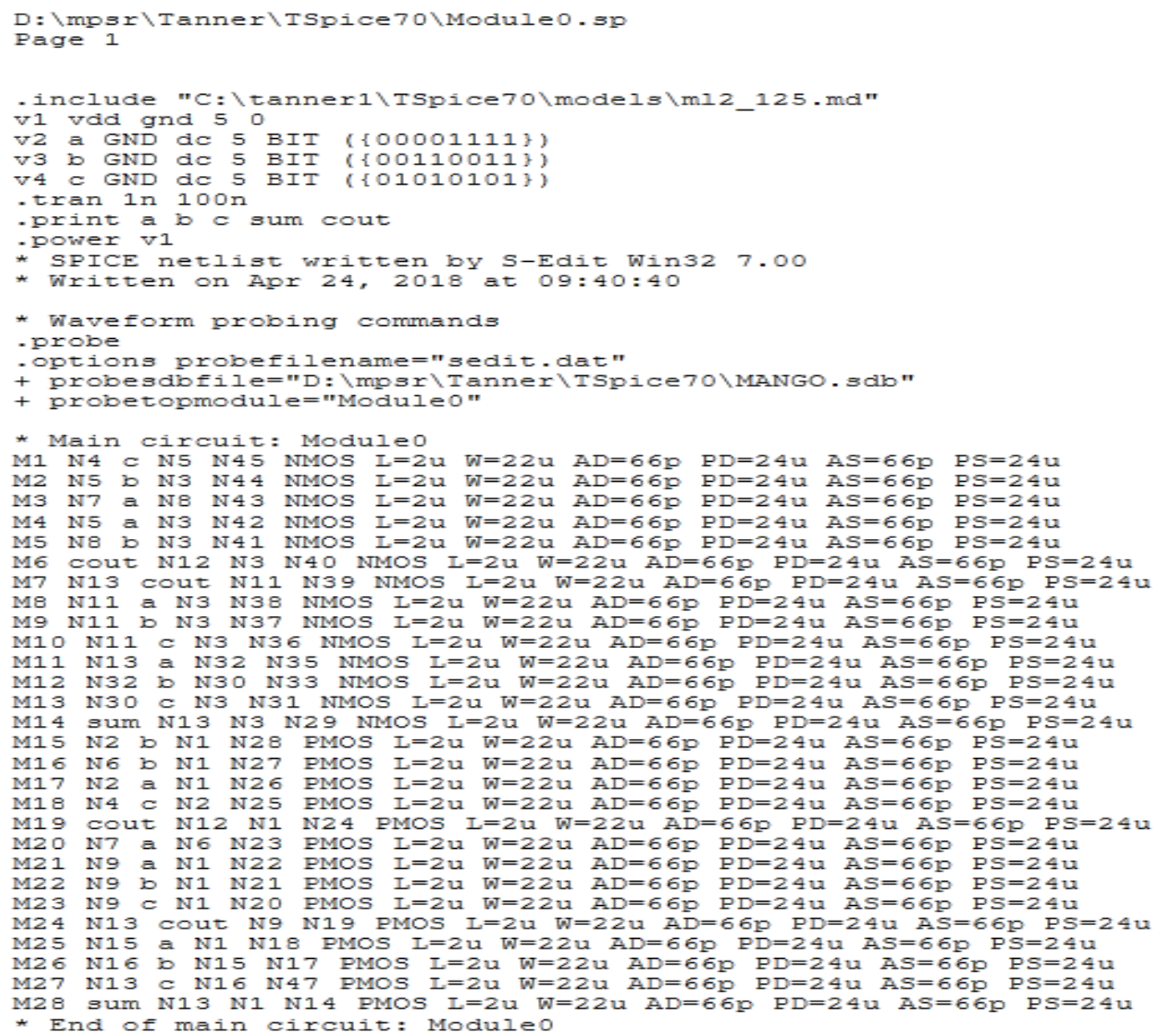


C. Power Analysis

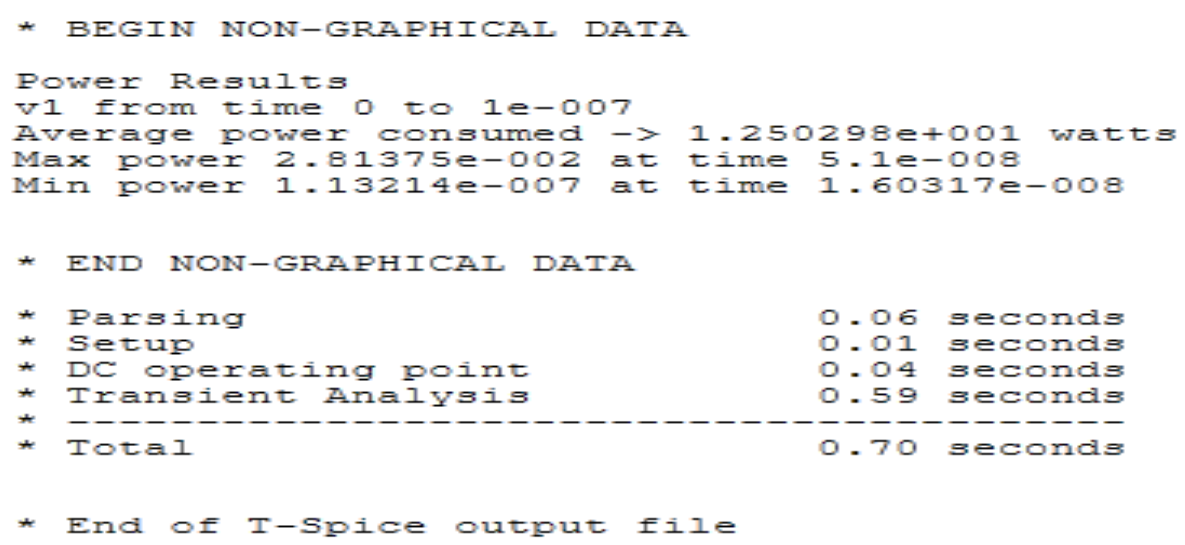

III CMOS Technology

The Complementary-symmetry Metal Oxide Semiconductor (CMOS) is a technology used for constructing integrated circuits [2]. The words "complementarysymmetry" refers to the typical design style with CMOS that uses complementary and symmetrical pairs of p-type and n-type metal oxide semiconductor field effect transistors (MOSFETs) for logic functions. Two important characteristics of CMOS devices are high noise immunity and low static power consumption[3]. Since one transistor of the pair is always off, the series combination draws significant power only momentarily during switching between on and off states.

A. Static and Dynamic Logic

1.Static Logic

The static, or steady-state, behavior of simple CMOS structures provides implementation of various logic function in the static logic circuit [4]. A typical static logic gate generates its output levels as long as the power supply is provided. It requires a large number of transistors to implement a function, that may cause a considerable time delay[5]. The schematic diagram of a static full adder is shown in Fig 3.

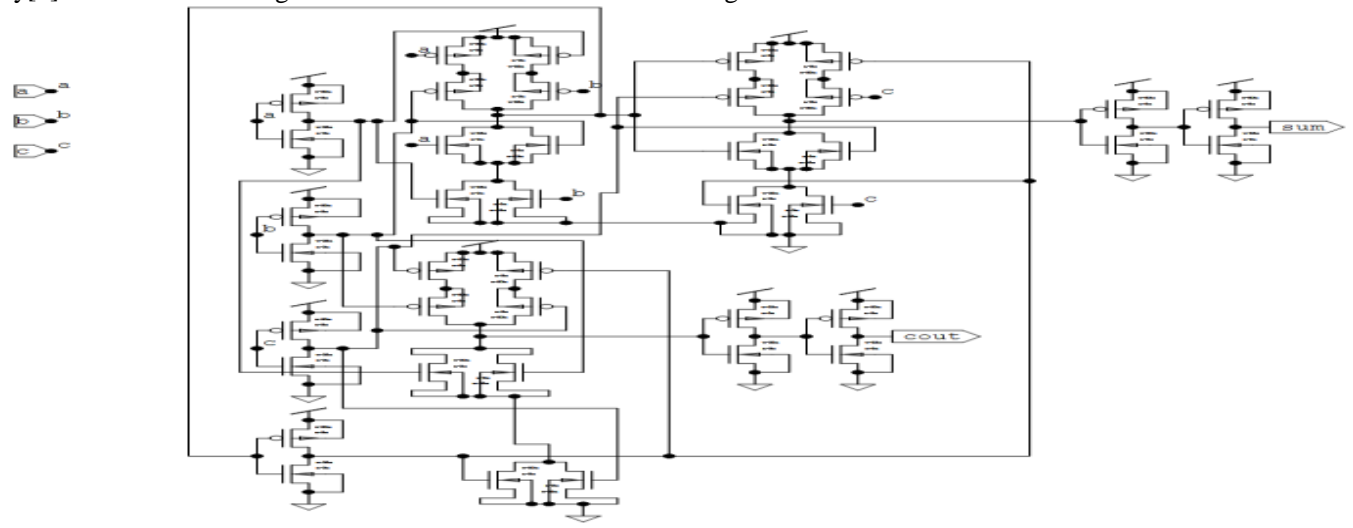

Fig 3: Schematic diagram for STATIC full adder

2. Netlist

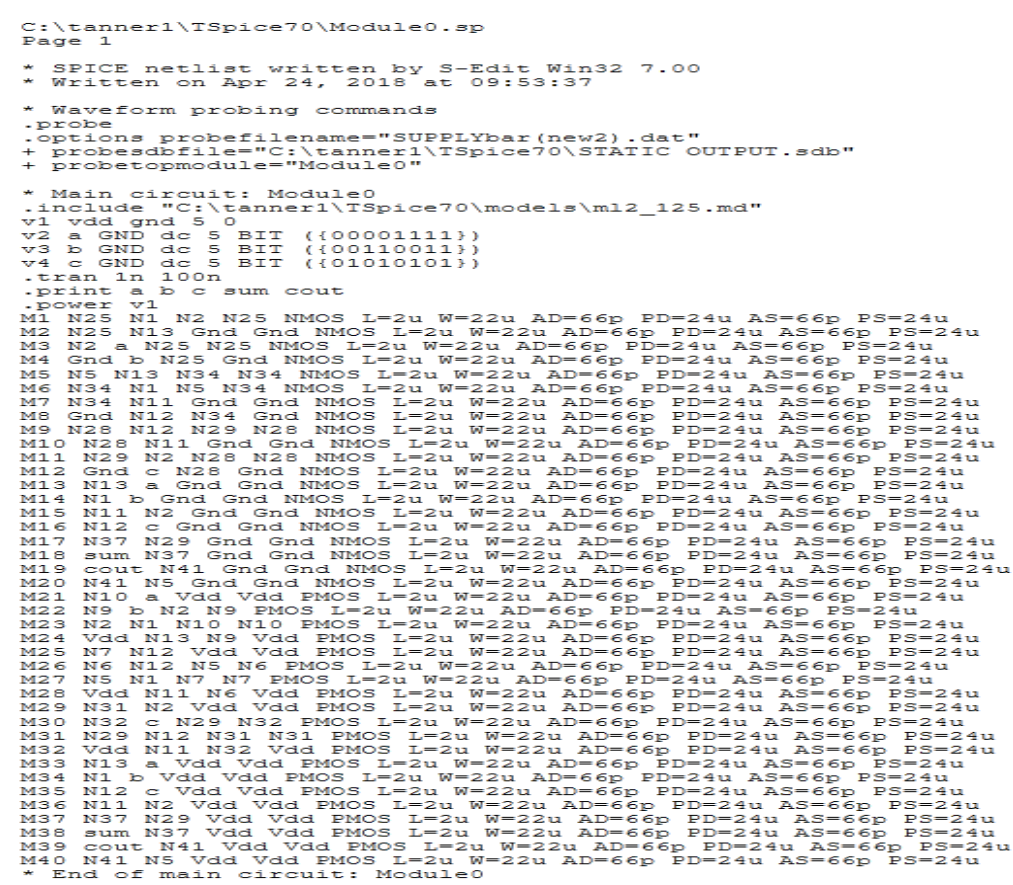


3. Power Analysis

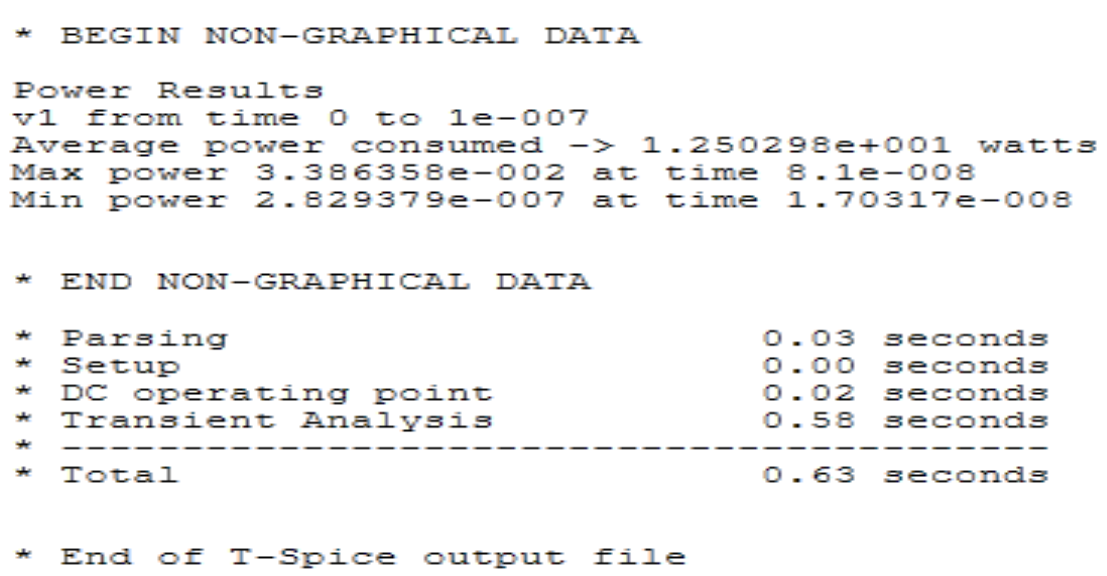

B.Dynamic Logic

Higher density and the higher performance of the digital implementations in the circuit where reduction of circuit delay and silicon area is a major objective of dynamic logic circuits offer several significant advantages over static logic circuits [6]. Fig.4 shows a generalized CMOS dynamic logic circuit. The operation of all dynamic logic gates depends on the temporary storage of charge in parasitic. This operational property should have the periodic updating of internal node voltage levels, since stored charge in capacitor cannot be retained indefinitely [7]. The dynamic logic circuit requires periodic clock signals to control the charge refreshing. The following dynamic CMOS circuit technique allows us to significantly reduce the number of transistors that are used to implement any logic function is introduced. The circuit that is based on first precharging involves evaluation of the output node capacitance and the output levels according to the applied inputs [8]. The precharge phase involves setting the circuit at a predefined initial state while the actual logic response is determined during the evaluation phase. Even though the Static CMOS offers a good performance it cannot keep up with dynamic logic styles in terms of propagation delay. The shorter delays is achieved by having the maximum tradeoff for increased power dissipation [9].

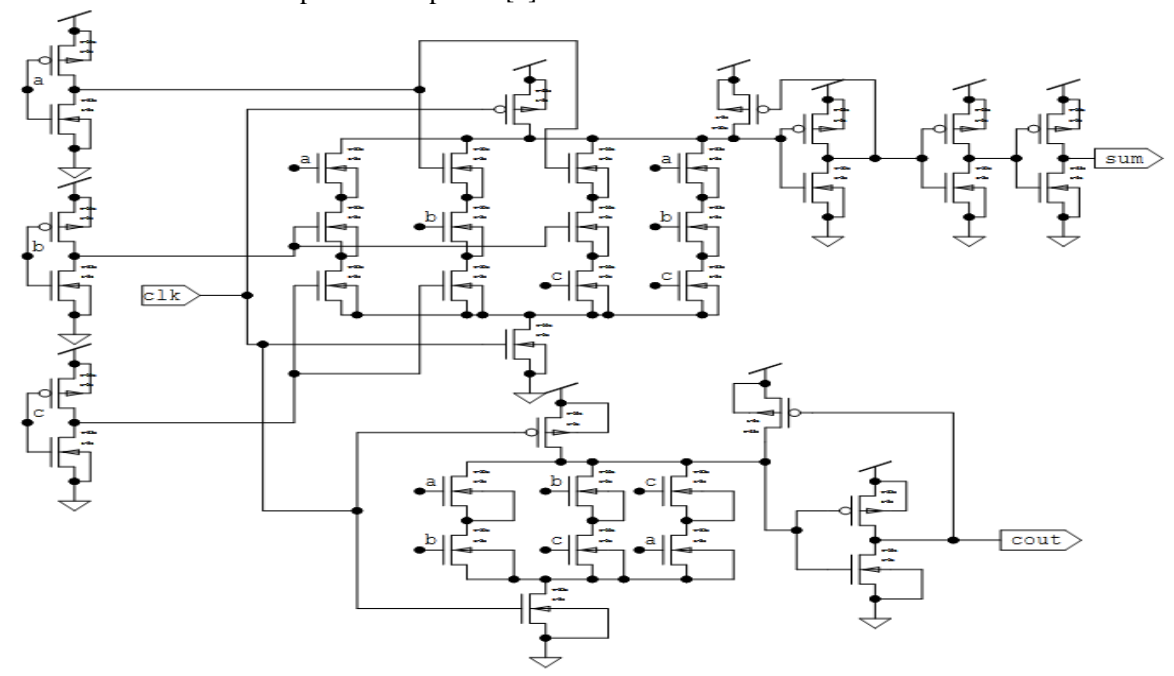

Fig 4: Schematic diagram for Dynamic Full Adder 1.Netlist 
2.Power Analysis

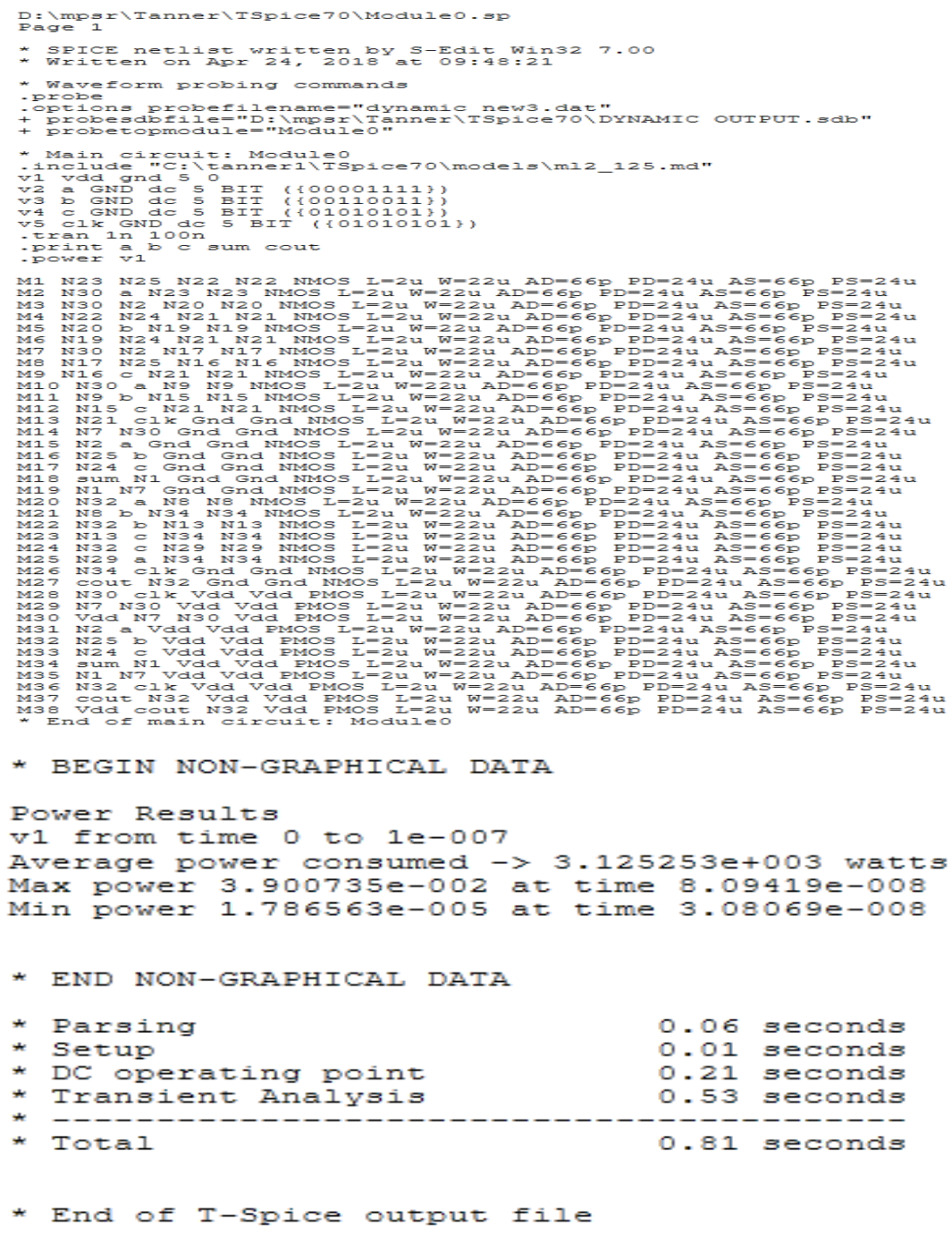

IV Interpolation

The principle of interpolation involves the construction of new data points set within the range of a discrete set of known data points[10]. It is often required to interpolate the value of that function for an intermediate value of the independent variable. The main problem related to interpolation is the approximation of a complicated function by a simple function. A few known data points from the original function can be used to create an interpolation based on a simpler function. When a simple function is used to estimate data points from the original, interpolation errors are usually present. The greater gain value depends on the problem domain and the interpolation method used.

V Fast Fourier Transform

The Fast Fourier transform, samples a signal over a certain period of time and divides into its frequency components. At distinct frequencies these frequency components are single sinusoidal oscillations that has their own amplitude and phase.

The computation of FFT algorithm involves two different transforms namely discrete Fourier transform (DFT) or its inverse (IFFT). The analysis of a Fourier transform involves the conversion of a signal from its original domain to a representation in the frequency domain and vice versa[11]. The transformations performed in FFT is rapidly computed by factorizing the DFT matrix into a product of sparse factors. The FFT involves the computation of the DFT and produces exactly the similar result when the DFT is being evaluated. The most important difference between DFT and FFT is that the latter is much faster. The DFT is defined by the formula,

where $\mathrm{x} 0, \ldots ., \mathrm{xN}-1$ be complex numbers.

VI Window Function

The Windowing technique of a simple waveform like cos $\omega t$ causes its Fourier transform to develop non-zero values at frequencies other than $\omega$. The leakage tends to be worst nearer to $\omega$ and minimum frequencies farthest from $\omega[12]$. The waveform that is brought under analysis comprises of two sinusoids with different frequencies, where the leakage has the ability to interfere from distinguishing them spectrally. The leakage from the stronger component can obscure the weaker one's presence only if the frequencies are dissimilar and one component is weaker. But if the frequencies are similar, leakage turns unresolvable even when the sinusoids are of equal strength.

A.Types Of Windowing Function

There are many windows proposed that approximate the desired characteristics. The basic window functions are listed below:

1. Rectangular Window

The rectangular window also called as the box car or Dirichlet window is the simplest window, that is equivalent to replacing all but the $\mathrm{N}$ values of a data sequence are replaced by zeros, making it to appear as though the waveform suddenly turns on and off: W(n) = 1. The FFT Analysis of a Full adder (Rectangular Window) is shown in Fig.5. The M point rectangular window, that corresponds to direct truncation of the Fourier series, has the weighted function as follows, 

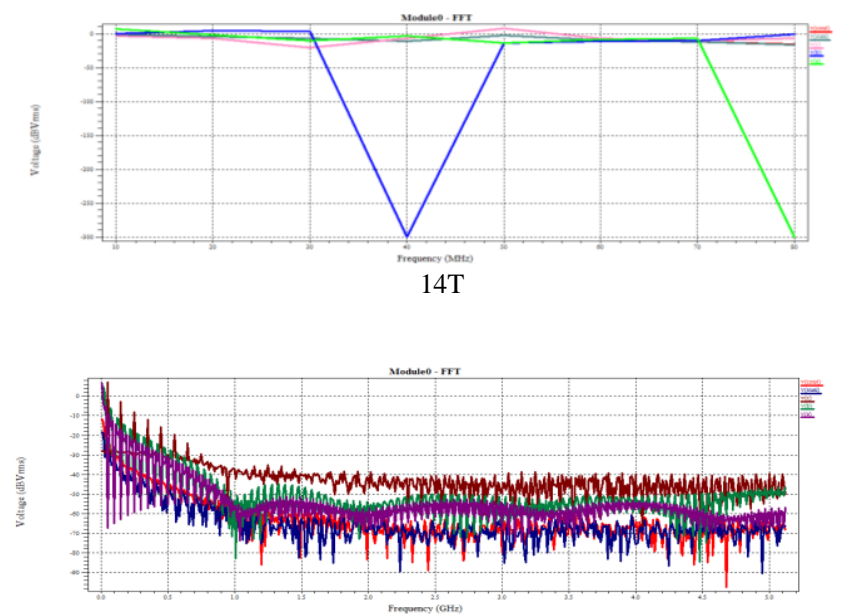

STATIC

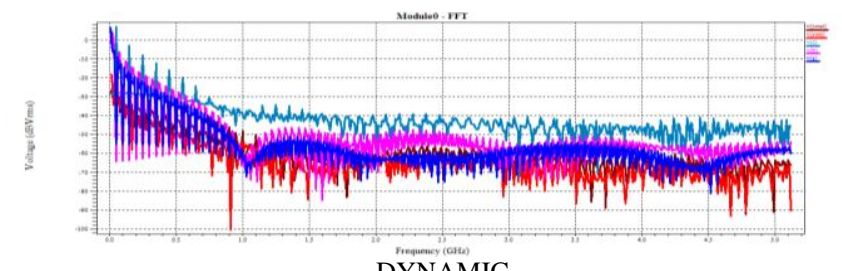

Fig 5: FFT Analysis of Full adder (Rectangular Window)

\section{Barlett Window}

Triangular windows are given by:

Where $\mathrm{L}$ can be $\mathrm{N}, \mathrm{N}+1$, or $\mathrm{N}-1$.

The latter is also known as Bartlett window[13]. All three definitions converge at large N. The triangular window is the 2nd order B-spline window and can be seen as the convolution of two half-sized rectangular windows, giving it twice the width of the regular windows. By tapering the rectangular window sequence linearly from the middle to the ends, we then obtain the M point triangular window[14]. The FFT Analysis of a Full adder (Barlett Window) is shown in Fig.6.
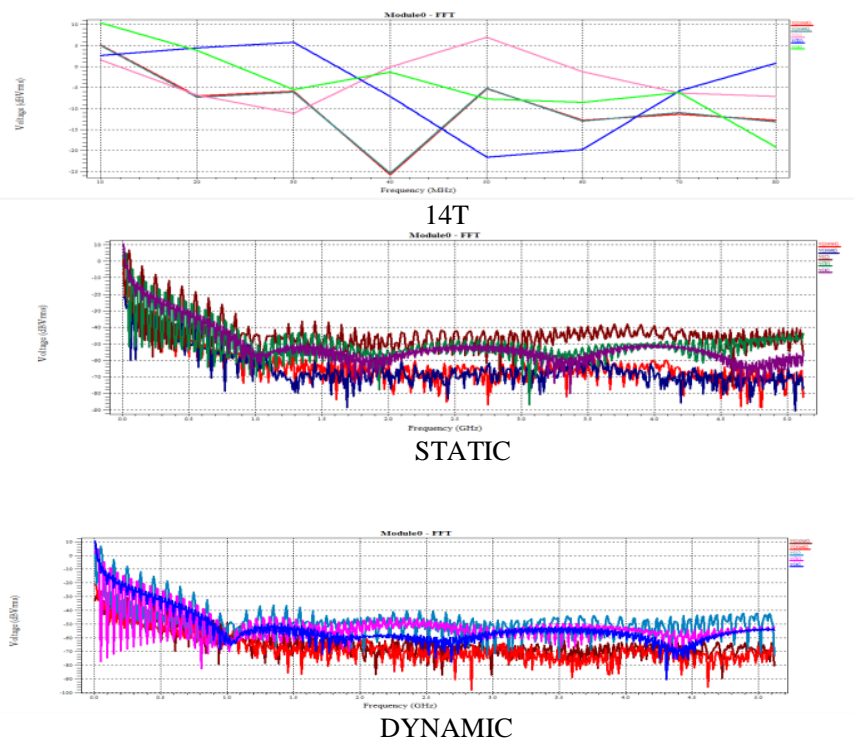

Fig 6: FFT Analysis of Full adder (Barlett Window)

\section{Hamming Window}

The hamming window is optimized in order to minimize the maximum that is the nearest side lobe, giving it a height of about one-fifth that of the Hann window[15]. It can be represented by,

$w(n)=\alpha-\beta \cos \left(\frac{2 \pi n}{N-1}\right)$ with $\alpha=0.54, \beta=1-\alpha=0.46$

instead of both the constants that is being equal to 1/2 in the Hann window. The FFT Analysis of a Full adder (Hamming Window) is shown in Fig.7.

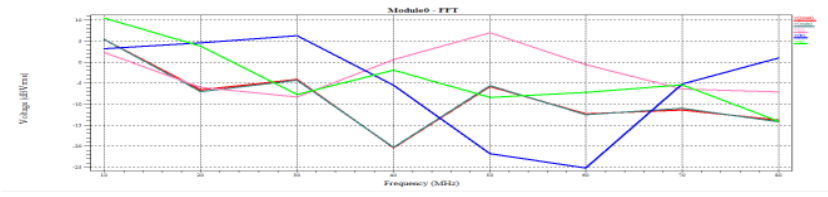

$14 \mathrm{~T}$

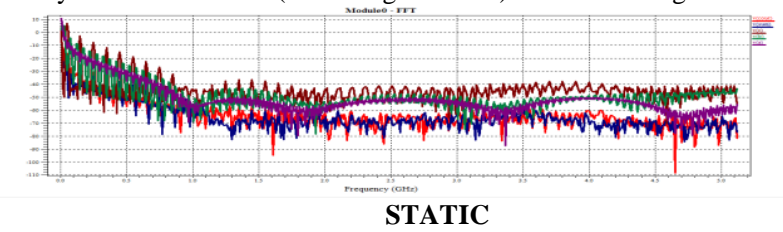

STATIC 


\section{Hanning Window}

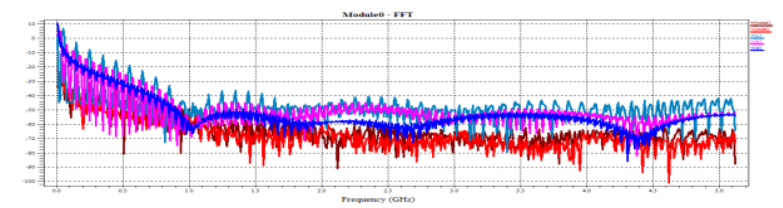

DYNAMIC

Fig 7: FFT Analysis of Full adder (Hamming Window)

The Hann window is also known as the Hanning window as it was named after Julius Von Hann and (for being similar in name and giving a form to the Hamming window), Von Hann and finally the raised cosine window is defined by,

$\mathrm{W}_{\mathrm{c}}(\mathrm{n})=0.5-0.5 \cos \left(\frac{2 \pi n}{M}-1\right)$, for $0 \leq n \leq(M-$

1)

o

otherwise

The FFT Analysis of a Full adder (Hanning Window) is shown in Fig.8.

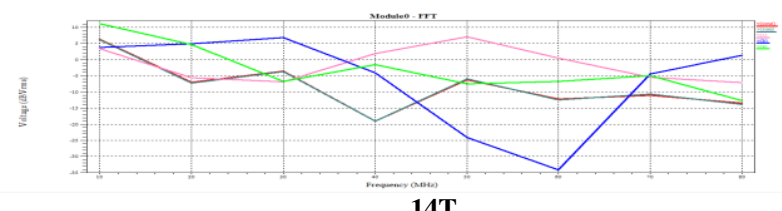

14T

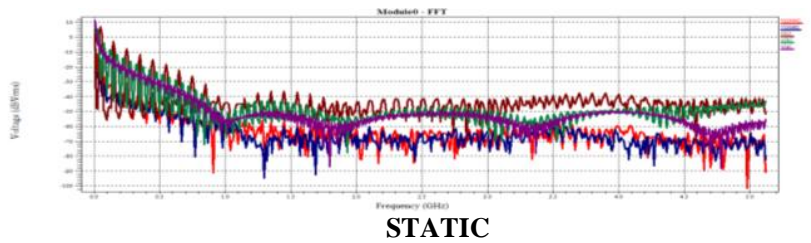

STATIC

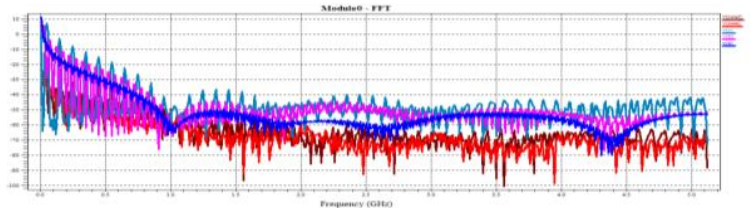

DYNAMIC

Fig 8: FFT Analysis of Full adder (Hanning Window)

\section{Blackman Harris Window}

The Blackman-Harris (BH) window is obtained by the straightforward generalization of the Hamming window[16]. The generalized Hamming family can be further constructed by using a summation of three shifted and scaled aliased-sinc-functions[17]. Then the Blackman-Harris window is obtained by adding still more shifted sinc functions:

$$
w_{B H}(n)=w_{R}(n) \sum_{l=0}^{L-1} \alpha_{l} \cos \left(l \Omega_{M} n\right),
$$

where $\mathrm{w}_{\mathrm{R}}(\mathrm{m})$ is the length $\mathrm{M}$ zero phase rectangular window. The corresponding window transform is given by,

$$
W_{B H}(\omega)=\sum_{k=-(L-1)}^{L-1} \alpha_{k} W_{R}\left(\omega+k \Omega_{M}\right),
$$

where $\mathrm{w}_{\mathrm{R}}(\mathrm{w})$ denotes the rectangular-window transform. The FFT Analysis of a Full adder (Blackman Harris Window) is shown in Fig.9.

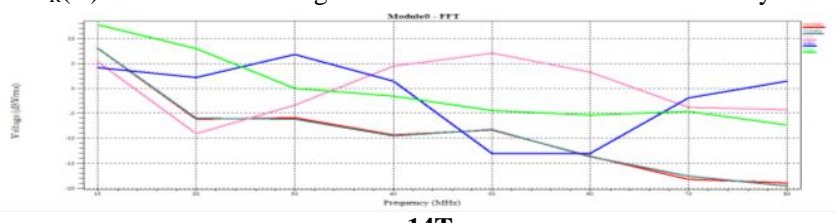

$14 \mathrm{~T}$

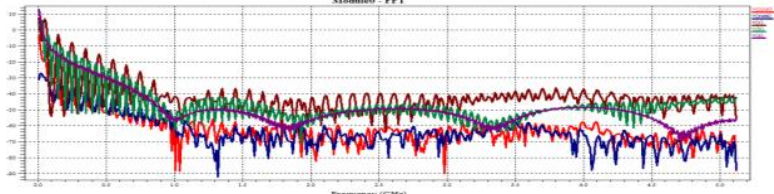

STATIC

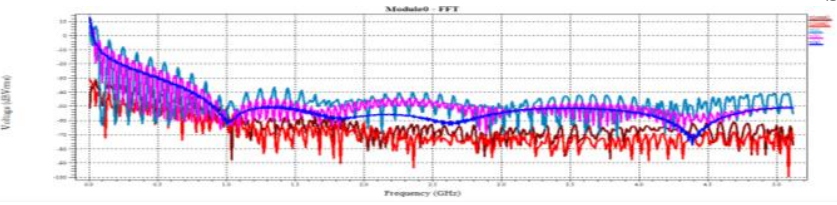

DYNAMIC

Fig 9: FFT Analysis of Full adder (Blackmann Harris Window)

\section{Blackmann Window}

The Blackman window is used along with the Kaiser, Hamming and the Blackman-Harris windows, and is considered as the most commonly used and the most popular windows[18]. Relatively high attenuation makes this window very convenient for almost all applications. The FFT Analysis of a Full adder (Blackman Window) is shown in Fig.10.The minimum stop band attenuation of a filter designed with this window amounts to 75dB[19]. The Blackman window coefficients are expressed as:

$$
w[n]=0.42-0.5 \cos \left(\frac{2 \pi n}{N-1}\right)+0.08 \cos \left(\frac{4 \pi n}{N-1}\right)
$$




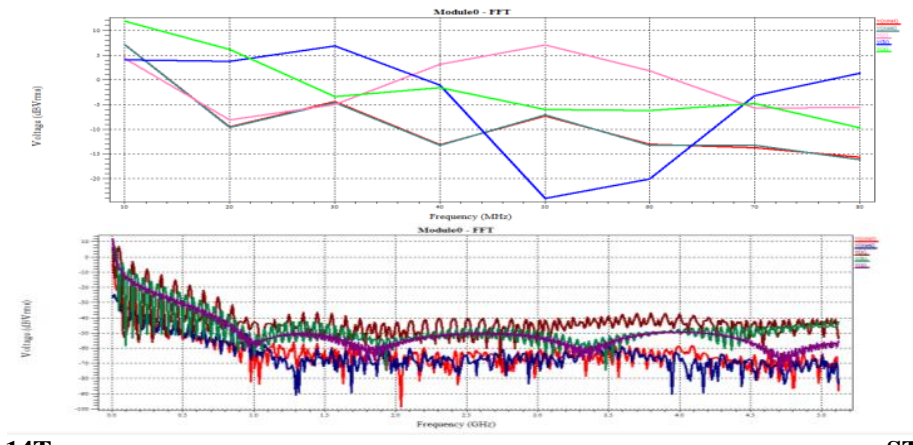

14T

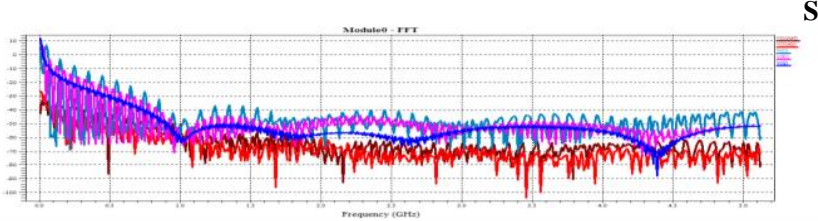

STATIC

\section{Welch Window}

DYNAMIC

Fig 10: FFT Analysis of Full adder (Blackmann Window)

The welch window is used for estimating the power of a signal at different frequencies i.e. it is an approach to estimate the spectral density[20]. This windowing method is mainly based on the concept of using periodogram spectrum estimates, that will result from converting a signal from the time domain to frequency domain. The main purpose of the window function $\mathrm{W}(\mathrm{N})$ is to reduce side-lobe level in the spectral density estimate, at the maxima of frequency resolution, as present in the case of sinusoidal spectrum analysis[21]. The FFT Analysis of a Full adder (Welch Window) is shown in Fig.11.

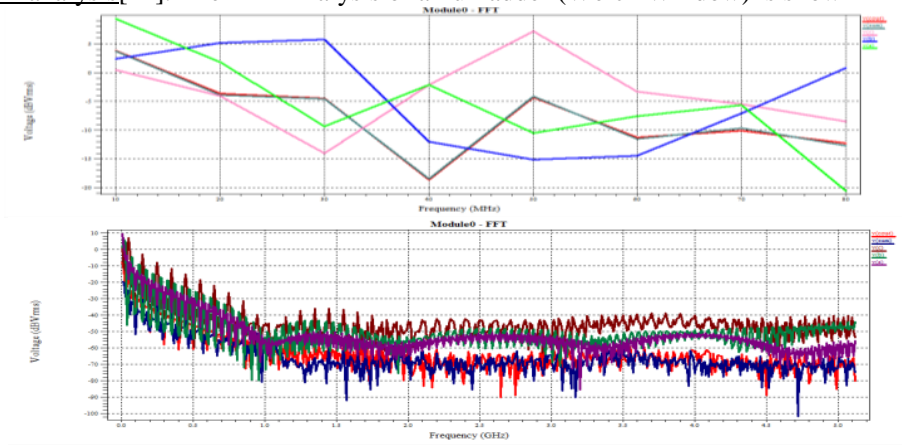

$14 \mathrm{~T}$

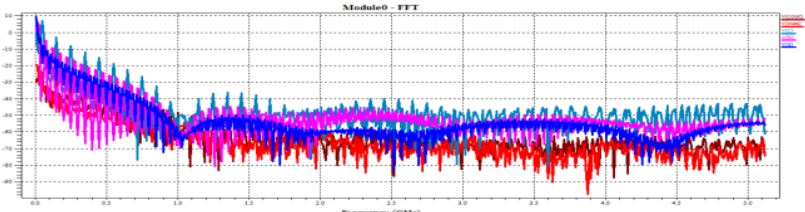

STATIC

DYNAMIC

Fig 11: FFT Analysis of Full adder (Welch Window)

8. Kaiser Bessel

The most important window among all windows is the Kaiser window [22]. The Kaiser window is a parametric window as it has an independent control parameter $\alpha$. The arbitrary specifications can be achieved in low pass (LP), high pass (HP), band pass (BP), and band stop (BS) filters by means of choosing the value of $\alpha$ and the filter length, N. The FFT Analysis of a Full adder (Kaiser Bessel Window) is shown in Fig.12[23]. The Kaiser window function is given by,

and $\mathrm{M}=(\mathrm{N}-1) / 2$. Its frequency spectrum is given by,

$$
\begin{gathered}
w_{\kappa}(n \pi)- \begin{cases}\frac{l_{0}(\beta)}{l_{0}(\alpha)} & \text { for }|n|=M \\
0 \text { othorwise }\end{cases} \\
\beta=\alpha \sqrt{1-\left(\frac{n}{M}\right)^{2}}, \quad l_{0}(X)=1+\sum_{k=1}^{\infty}\left[\frac{1}{k !}\left(\frac{X}{2}\right)^{k}\right]^{2}
\end{gathered}
$$

$$
W_{K}\left(e^{j \omega T}\right)=w_{K}(0)+2 \sum_{n=1}^{M} w_{K}(n T) \cos \omega n T
$$

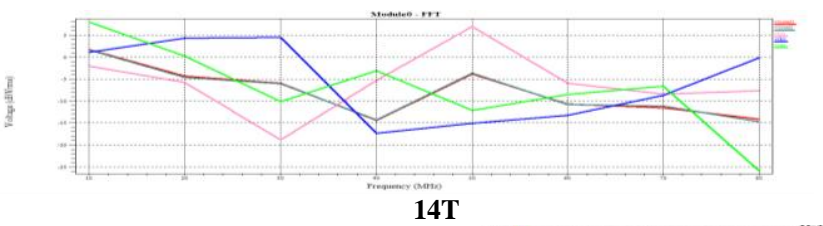

$14 \mathrm{~T}$

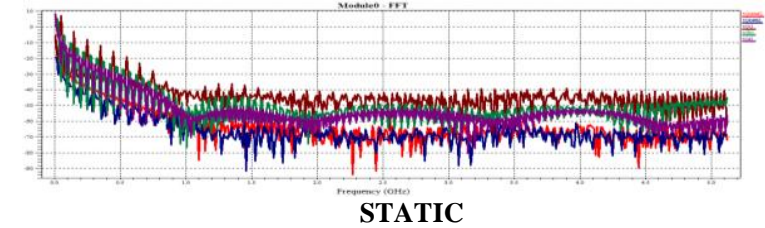

STATIC

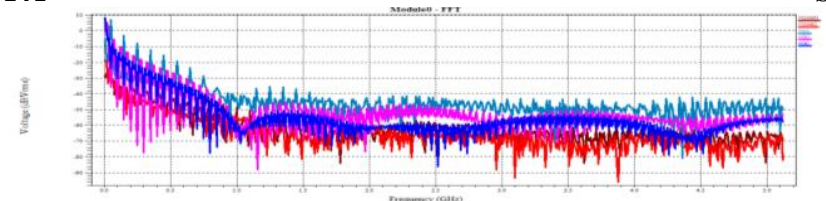

DYNAMIC

Fig 12: FFT Analysis of Full adder (Kaiser Bessel Window) 


\section{Gaussian Window}

The Fourier transform of a Gaussian is also a Gaussian if it is an eigen function of the Fourier Transform. As the Gaussian function extends to the value of infinity, it must be either truncated at the ends of the window, or it should be windowed with another zero-ended window[24]. The log of a Gaussian produces a parabola and hence it can be used for a quadratic interpolation in the process of frequency estimation. The FFT Analysis of a Full adder (Gaussian Window) is shown in Fig.13[25].

$$
\boldsymbol{w}(\boldsymbol{n})=e^{-\frac{1}{2}\left(\frac{n-(N-1) / 2}{\sigma(N-1) / 2}\right)^{2}}
$$

Here, the standard deviation of the Gaussian function is always $\sigma(N-1) / 2$ sampling periods.

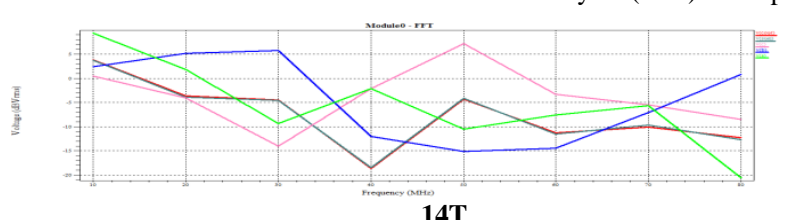

$14 \mathrm{~T}$

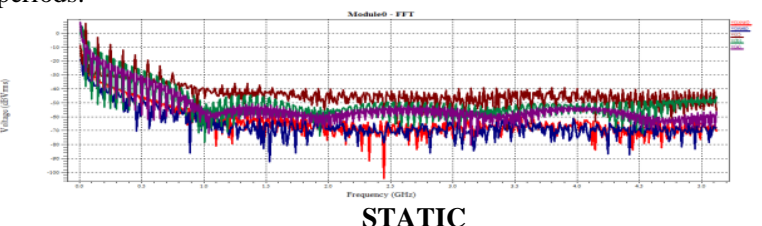

STATIC

VII

Fourier Transformation In Image

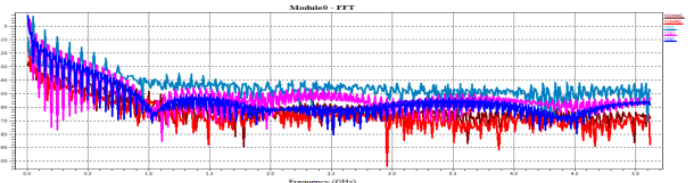

DYNAMIC

Fig 13: FFT Analysis of Full adder (Gaussiann Window)

The Fourier transform technique involves the decomposition of signals into their constituent waveforms in terms of their frequency, amplitude and phase domain [26]. Here, the waveforms are described in a circular form therefore the amplitude corresponds to the circle radius, and the frequency the angular velocity around the circumference and the phase, the starting angle on the circle [27]. The Fourier transforms follows the orthogonality property that serves as the critical property which allows different frequency sines and cosines to be combined yet give a signal with an mean of 0 .

Similarly, images can also be treated as signals where pixel intensity is considered as the signal amplitude and displacement in $\mathrm{X}$ and $\mathrm{Y}$ is considered as the frequency component [28]. Normally in images the information is not periodic in space, however the Fourier Transform can still be used for the decomposition of the image signal and can give more useful information. The convolution in real space is equivalent to multiplication in frequency space and the filtering of the image can be done for any high, low or orientated frequencies. Thus, the Fourier transform is a separable function and a Fast Fourier Transform of a 2D image signal can be performed by convolution of the image rows followed by the columns. This 2D FFT operation arranges the low frequency peaks at the corners of the image that are not convenient for filtering [29]. Noise figure (NF) or noise factor $(F)$ can be defined as the measures of degradation of the signal-to-noise ratio (SNR) that are mainly caused by the components in a radio-frequency (RF) signal chain. Here the performance of an amplifier or a radio receiver is always specified by a number, where the lower values indicates the better performance [30].

The FFT Transformation of images are shown in Fig.14.

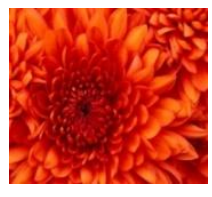

(a) Original Image

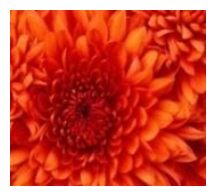

(d) $\mathrm{NF}=25 ; \mathrm{S}=10$

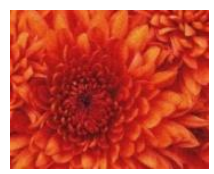

(g) $\mathrm{NF}=25 ; \mathrm{S}=75$

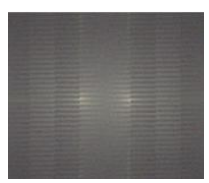

(b) FFT Transform

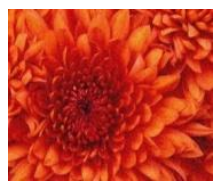

(e) $\mathrm{NF}=25 ; \mathrm{S}=25$

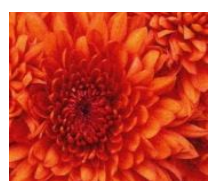

(h) $\mathrm{NF}=50 ; \mathrm{S}=25$

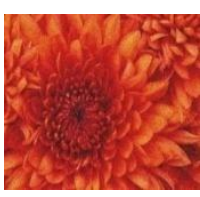

(c) $\mathrm{NF}=25 ; \mathrm{S}=50$

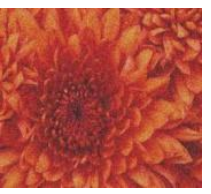

(f) $\mathrm{NF}=50 ; \mathrm{S}=50$

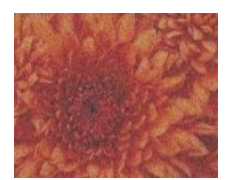

(i) $\mathrm{NF}=50 ; \mathrm{S}=100$

Fig 14: FFT Transformation of images(NF=Noise Factor and $\mathrm{S}=$ Strength $)$

\begin{tabular}{|c|c|c|}
\hline $\begin{array}{c}\text { CIRCUIT } \\
\text { NAME }\end{array}$ & $\begin{array}{c}\text { MAX } \\
\text { POWER }\end{array}$ & $\begin{array}{c}\text { MIN } \\
\text { POWER }\end{array}$ \\
\hline 14T & $0.28 \mathrm{mw}$ & $0.013 \mathrm{nw}$ \\
\hline STATIC & $0.33 \mathrm{mw}$ & $0.028 \mathrm{nw}$ \\
\hline DYNAMIC & $0.39 \mathrm{mw}$ & $0.17 \mu \mathrm{w}$ \\
\hline
\end{tabular}


In this paper, the fourier transform is performed for a $14 \mathrm{~T}$ transistor in static and dynamic styles with the help of tanner tool. The window function for each (static, dynamic, 14T) design styles is carried out for the interpolation 2 with the $\mathrm{N}$ value 10 is performed. The FFT Analysis of Full adder for each (Rectangular, Barlett, Hamming, Hanning, BlackmannHarris, Blackmann, Welch, Kaiser Bessel, Gaussian) windows is performed and the power is calculated [31]. The maximum power is found to be as $0.39 \mathrm{mw}$ and the minimum power obtained is $0.013 \mathrm{nw}$. Finally, the comparison is made between the powers of static and dynamic designing techniques. By the use of Fast Fourier Transformation (FFT), the transformation used in each window allows to evaluate the power at various interpolation rate. Thus, the scope lies in a reduced power consumption. The Noise Factor (NF) and the Strength (S) is calculated, and for the noise factor value 50, the strength attained has the value 100 . The future work involves the use of optimization techniques such as adiabatic logic, power gating to reduce the power consumption.

[1] D. Nayak, D.P. Acharya and K.K. Mahapatra, "Current starving the SRAM Cell: a strategy to improve cell stability and power".Circuits System and Signal Processing 2016.

[2] D. Nayak, D.P. Acharya and K.K. Mahapatra, "Power efficient design of a novel SRAM cell with higher write ability," in proceedings of IEEE India Conference (INDICON), pp. 1-6, Dec.2015

[3] N.Suvarna Parvathi Lakshmi, Mohd. Imaduddin and G. SanathKumar, "Design and Implementation of Sub Micron Level 10TFull Adder in ALU Using Cell Based and SOC Technology", International Journal of Emerging Technology and Advanced Engineering, vol. 4, no. 9, Sept. 2014.

[4] JohnRabaey, "Digital integrated circuits", Second Edison, 2003.

[5] M. Alioto and G. Palumbo, "Analysis and Comparison on Full Adder Block in Submicron Technology", IEEE transactions on very large scale integration (VLSI) systems, vol. 10, no. 6, pp 806-823, dec. 2002.

[6] H. Mahmoud and M. Bayoumi, "A 10-transistor low-power high speed full adder cell," in Proc. of ISCAS'99, pp. 43-46, June 1999,

[7] R. Shalem, E. John, and L. K. John, "A novel low power energy recovery full adder cell," in Proc. IEEE Great Lakes VLSI Symp., Feb.1999, pp. 380383.

[8] R. Zimmermann and W. Fichtner, "Low-power logic styles: CMOS versus pass-transistor logic,” IEEE J. Solid-State Circuits, vol. 32, pp.1079-1090, July 1997.

[9] V. G. Oklobdzija, M Soderstrand and B.Duchene "Development and Synthesis Method for Pass-Transistor Logic Family for High-Speed and Low Power CMOS" Proceedings of the 1995 IEEE 38th Midwest Symposium on Circuits and Systems, Rio de Janeiro, 1995.

[10] N. Weste and K. Eshraghian, "Principles of CMOSVLSI Design”, A System Perspective. Reading, MA: Addison-Wesley, 1993.Nan Zhuang and Haomin Wu, "A new design of the CMOS full adder "IEEE Journal of Solid State Circuits, Vol. 27, No.5, pp. 840-844, May 1992.

[11] R. ArunSekar, G. Naveen Balaji, A. Gautami, B. Sivasankari "High Efficient Carry Skip Adder in various Multiplier Structures" Advances in Natural and Applied Sciences (Annexure II), Vol. 10 Issue 14 (Special) (Oct 2016) pp: 193-197, ISSN: 1995-0772

[12] M. Srinivasaperumal, K. Boopathi Raja, G. Naveen Balaji, E. Christina Dally "Concurrent Node Recovery From Failure In Wireless Sensor-Actor Networks" KARI Research Journal, Vol. 1 Issue 4 (Oct - Dec 2016) pp: 28-33, ISSN: 2456-6136

[13] G. Naveen Balaji, V. Narayanan,V.S. Nivash "Low Power and High performance JK Flip - Flop using 45 nm Technology" International Journal of Engineering Research in Electronics and Communication Engineering (IJERECE) Vol 3, Issue 10, October 2016, pp:26-29, ISSN: $2394-6849$

[14] M. Srinivasaperumal, K. Boopathi Raja, G. Naveen Balaji, E. Christina Dally "Concurrent Node Recovery From Failure In Wireless Sensor-Actor Networks" Advances in Natural and Applied Sciences (Annexure II), Vol. 10 Issue 17 (Dec 2016) pp: 240-246, ISSN: 1995-0772

[15] G. Naveen Balaji, R. Prabha, E. Shanthini, J. Jayageetha, Mohand Lagha "Rapid low power Synchronous circuits using transmission gates" Advances in Natural and Applied Sciences (Annexure II), Vol. 10, Issue 17 (Dec 2016) pp: 287-291, ISSN: 1995-0772

[16] G. Naveen Balaji, S. Chenthur Pandian, D. Rajesh "Fast Test Pattern Generator using ATALANTA M 2.0" Asian Journal of Research in Social Sciences and Humanities (Annexure I) Vol. 7 No. 2 (Feb 2017) pp. 721-729 ISSN: 2249-7315 DOI: 10.5958/2249-7315.2017.00124.1

[17] G. Naveen Balaji, V. Aathira, K. Ambhikavathi, S. Geethiga, R. Havin "Low Power and High Speed Synchronous Circuits using Transmission Gates" Asian Journal of Research in Social Sciences and Humanities (Annexure I), Vol. 7 No. 2 (Feb 2017) pp. 713-720. ISSN: 2249-7315, DOI: 10.5958/22497315.2017.00123.X

[18] G. Naveen Balaji, S. Anusha, J. Ashwini “GPS Based Smart Navigation for Visually Impaired Using Bluetooth 3.0” Imperial Journal of Interdisciplinary Research (IJIR) Vol. 3, No. 3, 2017, pp. 773-776. ISSN: 2454-1362

[19] G. Naveen Balaji, D. Rajesh "Smart Vehicle Number Plate Detection System for Different Countries Using an Improved Segmentation Method" Imperial Journal of Interdisciplinary Research (IJIR) Vol. 3, No. 6, 2017, pp. 263-268. ISSN: 2454-1362

[20] G. Naveen Balaji, N.V. Hari Suriya, S. AnandVikash, R.Arun, S. Arun Kumar "Analysis of Various Liquid Components under Different Temperature and Density Constraints Pertaining To Fractional Distillation” Imperial Journal of Interdisciplinary Research (IJIR) Vol. 3, No. 6, 2017, pp. 664-669. ISSN: 2454-1362

[21] G. Naveen Balaji, D. Rajesh "Python Based Reverse Timing Algorithm for Human Brain Activity Using Color Psychology" International Journal of Indian Psychology, Vol. 4, No. 3, DIP: 18.01.111/20170403, pp: 79-86, ISSN 2348-5396

[22] G. Naveen Balaji, S. Chenthur Pandian, D. Rajesh "High Performance Triplex Adder Using CNTFET" International Journal of Trend in Scientific Research and Development, Vol.1, No. 5, pp: 368-373, ISSN 2456 - 6470

[23] G. Naveen Balaji, S. Chenthur Pandian, S. Giridharan, S. Shobana, J. Gayathri "Dynamic and Non-Linear Charge Transfer Through Opto-Deportation by Photovoltaic Cell" International Journal of Trend in Scientific Research and Development, Vol. 1, No. 5, pp: 486-492, ISSN 2456 - 6470

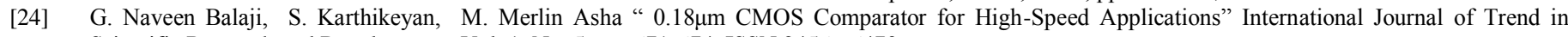
Scientific Research and Development, Vol. 1, No. 5, pp: 671-674, ISSN 2456 - 6470

[25] G. Naveen Balaji, K. Saravanan, R. Poorani, T. Vishnu Priya, R. Reka Raj "Advanced Security System using PIC through Bluetooth" International Journal of Trend in Scientific Research and Development, Vol. 1, No. 5, pp: 675-685, ISSN 2456 - 6470

[26] G. Naveen Balaji, N.V. Hari Suriya, S. Anand Vikash, S. Arun Kumar, R. Arun, "Gasoline Purity Meter Using Peripheral Interface Controller for Automobile Applications" International Journal of Engineering and Technical Research (IJETR), Vol. 7, No.10, pp:46-55, ISSN: 2321-0869

[27] G. Naveen Balaji, S.Chenthur Pandian, "Design for Testability of Kipbond Logic" "Perspectivas em Ciência da Informação" (Annexure - I), School of Information Science of the Federal University of Minas Gerais (UFMG), Vol. 22, No. SP.01, pp: 261-284, ISSN 1413-9936

[28] G. Naveen Balaji, V. Nandhini, S. Mithra, N. Priya , R. Naveena, "IOT Based Smart Crop Monitoring in Farm Land", Imperial Journal of Interdisciplinary Research (IJIR) Vol. 4, No. 1, pp: 88-92, ISSN: 2454-1362

[29] G. Naveen Balaji, N. V. Hari Suriya, "Effective Badminton Coaching Schema using PIC16F887 Microcontroller", International Journal of Trend in Scientific Research and Development, Vol. 2, No. 2, pp: 178 - 182, ISSN 2456 - 6470

[30] G. Naveen Balaji, S. Chenthur Pandian, "Power Analysis of a Transmission Gate based Scan Flip Flop", International Journal of Engineering Research in Electronics and Communication Engineering (IJERECE) Vol 4, Issue 11, November 2017, pp:48-53, ISSN: 2394-6849

[31] P.Malini, T.Poovika, P.Shanmugavadivu, G.Naveen Balaji, "Design of High Speed 8 Bit Carry Look Ahead Logic for Arithmetic Operations", International Journal of Engineering Research in Electronics and Communication Engineering (IJERECE) Vol 4, Issue 12, December 2017, pp:59-62, ISSN: 2394-6849 